\title{
ON STABLE ENTIRE SOLUTIONS OF SEMI-LINEAR ELLIPTIC EQUATIONS WITH WEIGHTS
}

\author{
CRAIG COWAN AND MOSTAFA FAZLY
}

(Communicated by James E. Colliander)

\begin{abstract}
We are interested in the existence versus non-existence of nontrivial stable sub- and super-solutions of

$$
-\operatorname{div}\left(\omega_{1} \nabla u\right)=\omega_{2} f(u) \quad \text { in } \mathbb{R}^{N},
$$

with positive smooth weights $\omega_{1}(x), \omega_{2}(x)$. We consider the cases $f(u)=$ $e^{u}, u^{p}$ where $p>1$ and $-u^{-p}$ where $p>0$. We obtain various non-existence results which depend on the dimension $N$ and also on $p$ and the behaviour of $\omega_{1}, \omega_{2}$ near infinity. Also the monotonicity of $\omega_{1}$ is involved in some results. Our methods here are the methods developed by Farina. We examine a specific class of weights $\omega_{1}(x)=\left(|x|^{2}+1\right)^{\frac{\alpha}{2}}$ and $\omega_{2}(x)=\left(|x|^{2}+1\right)^{\frac{\beta}{2}} g(x)$, where $g(x)$ is a positive function with a finite limit at $\infty$. For this class of weights, non-existence results are optimal. To show the optimality we use various generalized Hardy inequalities.
\end{abstract}

\section{INTRODUCTION AND MAIN RESULTS}

In this note we are interested in the existence versus non-existence of stable suband super-solutions of equations of the form

$$
-\operatorname{div}\left(\omega_{1}(x) \nabla u\right)=\omega_{2}(x) f(u) \quad \text { in } \mathbb{R}^{N},
$$

where $f(u)$ is one of the following non-linearities: $e^{u}, u^{p}$ where $p>1$ and $-u^{-p}$ where $p>0$. We assume that $\omega_{1}(x)$ and $\omega_{2}(x)$, which we call weights, are smooth positive functions (we allow $\omega_{2}$ to be zero at say a point) and which satisfy various growth conditions at $\infty$. Recall that we say that a solution $u$ of $-\Delta u=f(u)$ in $\mathbb{R}^{N}$ is stable provided that

$$
\int f^{\prime}(u) \psi^{2} \leq \int|\nabla \psi|^{2}, \quad \forall \psi \in C_{c}^{2}
$$

where $C_{c}^{2}$ is the set of $C^{2}$ functions defined on $\mathbb{R}^{N}$ with compact support. Note that the stability of $u$ is just saying that the second variation at $u$ of the energy

Received by the editors February 3, 2011.

2010 Mathematics Subject Classification. Primary 35B08; Secondary 35J61, 35A01.

Key words and phrases. Semi-linear elliptic equations, Hardy's inequality, stable solutions.

This work is supported by a University Graduate Fellowship and is part of the second author's Ph.D. dissertation in preparation under the supervision of N. Ghoussoub. 
associated with the equation is non-negative. In our setting this becomes: We say that a $C^{2}$ sub/super-solution $u$ of (1.1) is stable provided that

$$
\int \omega_{2} f^{\prime}(u) \psi^{2} \leq \int \omega_{1}|\nabla \psi|^{2} \quad \forall \psi \in C_{c}^{2}
$$

One should note that (1.1) can be rewritten as

$$
-\Delta u+\nabla \gamma(x) \cdot \nabla u=\omega_{2} / \omega_{1} f(u) \quad \text { in } \mathbb{R}^{N},
$$

where $\gamma=-\log \left(\omega_{1}\right)$, and on occasion we shall take this point of view.

Remark 1. Note that if $\omega_{1}$ has enough integrability, then it is immediate that if $u$ is a stable solution of (1.1) we have $\int \omega_{2} f^{\prime}(u)=0$ (provided $f$ is increasing). To see this let $0 \leq \psi \leq 1$ be supported in a ball of radius $2 R$ centered at the origin $\left(B_{2 R}\right)$ with $\psi=1$ on $B_{R}$ and such that $|\nabla \psi| \leq \frac{C}{R}$, where $C>0$ is independent of $R$. Putting this $\psi$ into (1.2) one obtains

$$
\int_{B_{R}} \omega_{2} f^{\prime}(u) \leq \frac{C}{R^{2}} \int_{R<|x|<2 R} \omega_{1},
$$

and so if the right-hand side goes to zero as $R \rightarrow \infty$ we have the desired result.

The existence versus non-existence of stable solutions of $-\Delta u=f(u)$ in $\mathbb{R}^{N}$ or $-\Delta u=g(x) f(u)$ in $\mathbb{R}^{N}$ is now quite well understood; see [3, 4, 7, 8, 9, 10, 11, 1, 5, 6]. We remark that some of these results are examining the case where $\Delta$ is replaced with $\Delta_{p}$ (the $p$-Laplacian), and also in many cases the authors are interested in finite Morse index solutions or solutions which are stable outside a compact set. Much of the interest in these Liouville type theorems stems from the fact that the non-existence of a stable solution is related to the existence of a priori estimates for stable solutions of a related equation on a bounded domain.

In 12 equations similar to $-\Delta u=|x|^{\alpha} u^{p}$ were examined on the unit ball in $\mathbb{R}^{N}$ with zero Dirichlet boundary conditions. There it was shown that for $\alpha>0$ one can obtain positive solutions for $p$ supercritical with respect to Sobolev embedding and so one can observe that the term $|x|^{\alpha}$ is restoring some compactness. A similar feature happens for equations of the form

$$
-\Delta u=|x|^{\alpha} f(u) \quad \text { in } \mathbb{R}^{N} ;
$$

the value of $\alpha$ can vastly alter the existence versus non-existence of a stable solution; see [5, 1, 6, 8, 7. 
We now come to our main results and for this we need to define a few quantities:

$$
\begin{aligned}
I_{G} & :=R^{-4 t-2} \int_{R<|x|<2 R} \frac{\omega_{1}^{2 t+1}}{\omega_{2}^{2 t}} d x, \\
J_{G} & :=R^{-2 t-1} \int_{R<|x|<2 R} \frac{\left|\nabla \omega_{1}\right|^{2 t+1}}{\omega_{2}^{2 t} d x,} \\
I_{L} & :=R^{\frac{-2(2 t+p-1)}{p-1}} \int_{R<|x|<2 R}\left(\frac{w_{1}^{p+2 t-1}}{w_{2}^{2 t}}\right)^{\frac{1}{p-1}} d x, \\
J_{L} & :=R^{-\frac{p+2 t-1}{p-1}} \int_{R<|x|<2 R}\left(\frac{\left|\nabla w_{1}\right|^{p+2 t-1}}{w_{2}^{2 t}}\right)^{\frac{1}{p-1}} d x, \\
I_{M} & :=R^{-2 \frac{p+2 t+1}{p+1}} \int_{R<|x|<2 R}\left(\frac{w_{1}^{p+2 t+1}}{w_{2}^{2 t}}\right)^{\frac{1}{p+1}} d x, \\
J_{M} & :=R^{-\frac{p+2 t+1}{p+1}} \int_{R<|x|<2 R}\left(\frac{\left|\nabla w_{1}\right|^{p+2 t+1}}{w_{2}^{2 t}}\right)^{\frac{1}{p+1}} d x .
\end{aligned}
$$

The three equations we examine are

$$
\begin{array}{ccc}
-\operatorname{div}\left(\omega_{1} \nabla u\right)=\omega_{2} e^{u} & \text { in } \mathbb{R}^{N} & (G), \\
-\operatorname{div}\left(\omega_{1} \nabla u\right)=\omega_{2} u^{p} & \text { in } \mathbb{R}^{N} & (L), \\
-\operatorname{div}\left(\omega_{1} \nabla u\right)=-\omega_{2} u^{-p} & \text { in } \mathbb{R}^{N} & (M),
\end{array}
$$

where we restrict $(L)$ to the case $p>1$ and $(M)$ to $p>0$. By a solution we always mean a $C^{2}$ solution. We now come to our main results in terms of abstract $\omega_{1}$ and $\omega_{2}$. We remark that our approach to non-existence of stable solutions is the approach due to Farina; see [9, 10, 4].

\section{Theorem 1.1.}

(1) There is no stable sub-solution of $(G)$ if $I_{G}, J_{G} \rightarrow 0$ as $R \rightarrow \infty$ for some $0<t<2$.

(2) There is no positive stable sub-solution (super-solution) of ( $L)$ if $I_{L}, J_{L} \rightarrow 0$ as $R \rightarrow \infty$ for some $p-\sqrt{p(p-1)}<t<p+\sqrt{p(p-1)}\left(0<t<\frac{1}{2}\right)$.

(3) There is no positive stable super-solution of (M) if $I_{M}, J_{M} \rightarrow 0$ as $R \rightarrow \infty$ for some $0<t<p+\sqrt{p(p+1)}$.

If we assume that $\omega_{1}$ has some monotonicity we can do better. We will assume that the monotonicity conditions are satisfied for large $x$ but really all one needs is for it to be satisfied on a suitable sequence of annuli.

\section{Theorem 1.2.}

(1) There is no stable sub-solution of $(G)$ with $\nabla \omega_{1}(x) \cdot x \leq 0$ for large $x$ if $I_{G} \rightarrow 0$ as $R \rightarrow \infty$ for some $0<t<2$.

(2) There is no positive stable sub-solution of $(L)$ provided $I_{L} \rightarrow 0$ as $R \rightarrow \infty$ for either:

- some $1 \leq t<p+\sqrt{p(p-1)}$ and $\nabla \omega_{1}(x) \cdot x \leq 0$ for large $x$ or

- some $p-\sqrt{p(p-1)}<t \leq 1$ and $\nabla \omega_{1}(x) \cdot x \geq 0$ for large $x$.

There is no positive super-solution of $(L)$ provided $I_{L} \rightarrow 0$ as $R \rightarrow \infty$ for some $0<t<\frac{1}{2}$ and $\nabla \omega_{1}(x) \cdot x \leq 0$ for large $x$. 
(3) There is no positive stable super-solution of $(M)$ provided $I_{M} \rightarrow 0$ as $R \rightarrow$ $\infty$ for some $0<t<p+\sqrt{p(p+1)}$.

Corollary 1. Suppose $\omega_{1} \leq C \omega_{2}$ for large $x, \omega_{2} \in L^{\infty}, \nabla \omega_{1}(x) \cdot x \leq 0$ for large $x$.

(1) There is no stable sub-solution of $(G)$ if $N \leq 9$.

(2) There is no positive stable sub-solution of $(L)$ if

$$
N<2+\frac{4}{p-1}(p+\sqrt{p(p-1)}) .
$$

(3) There is no positive stable super-solution of $(M)$ if

$$
N<2+\frac{4}{p+1}(p+\sqrt{p(p+1)}) .
$$

If one takes $\omega_{1}=\omega_{2}=1$ in the above corollary, the results obtained for $(G)$ and $(L)$, and for some values of $p$ in $(M)$, are optimal; see [9, 10, 8].

We now drop all monotonicity conditions on $\omega_{1}$.

Corollary 2. Suppose $\omega_{1} \leq C \omega_{2}$ for large $x, \omega_{2} \in L^{\infty},\left|\nabla \omega_{1}\right| \leq C \omega_{2}$ for large $x$.

(1) There is no stable sub-solution of $(G)$ if $N \leq 4$.

(2) There is no positive stable sub-solution of $(L)$ if

$$
N<1+\frac{2}{p-1}(p+\sqrt{p(p-1)}) .
$$

(3) There is no positive super-solution of $(M)$ if

$$
N<1+\frac{2}{p+1}(p+\sqrt{p(p+1)}) .
$$

Some of the conditions on $\omega_{i}$ in Corollary 2 seem somewhat artificial. If we shift over to the advection equation (and we take $\omega_{1}=\omega_{2}$ for simplicity)

$$
-\Delta u+\nabla \gamma \cdot \nabla u=f(u),
$$

the conditions on $\gamma$ become: $\gamma$ is bounded from below and has a bounded gradient.

In what follows we examine the case where $\omega_{1}(x)=\left(|x|^{2}+1\right)^{\frac{\alpha}{2}}$ and $\omega_{2}(x)=$ $g(x)\left(|x|^{2}+1\right)^{\frac{\beta}{2}}$, where $g(x)$ is positive, except at say a point, and smooth, and where $\lim _{|x| \rightarrow \infty} g(x)=C \in(0, \infty)$. For this class of weights we can essentially obtain optimal results.

Theorem 1.3. Take $\omega_{1}$ and $\omega_{2}$ as above.

(1) If $N+\alpha-2<0$, then there is no stable sub-solution for $(G),(L)$ (here we require it to be positive), and in the case of $(M)$ there is no positive stable super-solution. This case is the trivial case; see Remark 1.

Assumption. For the remaining cases we assume that $N+\alpha-2>0$.

(2) If $N+\alpha-2<4(\beta-\alpha+2)$, then there is no stable sub-solution for $(G)$.

(3) If $N+\alpha-2<\frac{2(\beta-\alpha+2)}{p-1}(p+\sqrt{p(p-1)})$, then there is no positive stable sub-solution of $(L)$.

(4) If $N+\alpha-2<\frac{2(\beta-\alpha+2)}{p+1}(p+\sqrt{p(p+1)})$, then there is no positive stable super-solution of $(M)$. 
(5) Furthermore, (2), (3), (4) are optimal in the sense that if $N+\alpha-2>$ 0 and the remaining inequality is not satisfied (and in addition we assume we do not have equality in the inequality), then we can find a suitable function $g(x)$ which satisfies the above properties and a stable sub-/super-solution $u$ for the appropriate equation.

Remark 2. Many of the above results can be extended to the case of equality in either $N+\alpha-2 \geq 0$ or the other inequality which depends on the equation we are examining. We omit the details because one cannot prove the results in a unified way.

In showing that an explicit solution is stable we will need the weighted Hardy inequality given in [2].

Lemma 1. Suppose $E>0$ is a smooth function. Then one has

$$
\left(\tau-\frac{1}{2}\right)^{2} \int E^{2 \tau-2}|\nabla E|^{2} \phi^{2}+\left(\frac{1}{2}-\tau\right) \int(-\Delta E) E^{2 \tau-1} \phi^{2} \leq \int E^{2 \tau}|\nabla \phi|^{2},
$$

for all $\phi \in C_{c}^{\infty}\left(\mathbb{R}^{N}\right)$ and $\tau \in \mathbb{R}$.

By picking an appropriate function $E$ this gives

Corollary 3. For all $\phi \in C_{c}^{\infty}$ and $t, \alpha \in \mathbb{R}$, we have

$$
\begin{aligned}
\int\left(1+|x|^{2}\right)^{\frac{\alpha}{2}}|\nabla \phi|^{2} \geq & \left(t+\frac{\alpha}{2}\right)^{2} \int|x|^{2}\left(1+|x|^{2}\right)^{-2+\frac{\alpha}{2}} \phi^{2} \\
& +\left(t+\frac{\alpha}{2}\right) \int\left(N-2(t+1) \frac{|x|^{2}}{1+|x|^{2}}\right)\left(1+|x|^{2}\right)^{-1+\frac{\alpha}{2}} \phi^{2} .
\end{aligned}
$$

\section{Proof of main Results}

Proof of Theorem 1.1. (1) Suppose $u$ is a stable sub-solution of $(G)$ with $I_{G}, J_{G} \rightarrow$ 0 as $R \rightarrow \infty$ and let $0 \leq \phi \leq 1$ denote a smooth compactly supported function. Put $\psi:=e^{t u} \phi$ into (1.2), where $0<t<2$, to arrive at

$$
\begin{aligned}
\int \omega_{2} e^{(2 t+1) u} \phi^{2} \leq & t^{2} \int \omega_{1} e^{2 t u}|\nabla u|^{2} \phi^{2} \\
& +\int \omega_{1} e^{2 t u}|\nabla \phi|^{2}+2 t \int \omega_{1} e^{2 t u} \phi \nabla u \cdot \nabla \phi .
\end{aligned}
$$

Now multiply $(G)$ by $e^{2 t u} \phi^{2}$ and integrate by parts to arrive at

$$
2 t \int \omega_{1} e^{2 t u}|\nabla u|^{2} \phi^{2} \leq \int \omega_{2} e^{(2 t+1) u} \phi^{2}-2 \int \omega_{1} e^{2 t u} \phi \nabla u \cdot \nabla \phi,
$$

and now if one equates like terms, one arrives at

$$
\begin{aligned}
\frac{(2-t)}{2} \int \omega_{2} e^{(2 t+1) u} \phi^{2} \leq & \int \omega_{1} e^{2 t u}\left(|\nabla \phi|^{2}-\frac{\Delta \phi}{2}\right) d x \\
& -\frac{1}{2} \int e^{2 t u} \phi \nabla \omega_{1} \cdot \nabla \phi .
\end{aligned}
$$

Now substitute $\phi^{m}$ into this inequality for $\phi$ where $m$ is a large integer to obtain

$$
\begin{gathered}
\frac{(2-t)}{2} \int \omega_{2} e^{(2 t+1) u} \phi^{2 m} \leq C_{m} \int \omega_{1} e^{2 t u} \phi^{2 m-2}\left(|\nabla \phi|^{2}+\phi|\Delta \phi|\right) d x \\
-D_{m} \int e^{2 t u} \phi^{2 m-1} \nabla \omega_{1} \cdot \nabla \phi
\end{gathered}
$$


where $C_{m}$ and $D_{m}$ are positive constants just depending on $m$. We now estimate the terms on the right, but we mention that when one assumes the appropriate monotonicity on $\omega_{1}$, it is the last integral on the right which one is able to drop:

$$
\begin{aligned}
\int \omega_{1} e^{2 t u} \phi^{2 m-2}|\nabla \phi|^{2}= & \int \omega_{2}^{\frac{2 t}{2 t+1}} e^{2 t u} \phi^{2 m-2} \frac{\omega_{1}}{\omega_{2}^{\frac{2 t}{2 t+1}}}|\nabla \phi|^{2} \\
\leq & \left(\int \omega_{2} e^{(2 t+1) u} \phi^{(2 m-2) \frac{(2 t+1)}{2 t}} d x\right)^{\frac{2 t}{2 t+1}} \\
& \times\left(\int \frac{\omega_{1}^{2 t+1}}{\omega_{2}^{2 t}}|\nabla \phi|^{2(2 t+1)}\right)^{\frac{1}{2 t+1}} .
\end{aligned}
$$

Now, for fixed $0<t<2$ we can take $m$ large enough so that $(2 m-2) \frac{(2 t+1)}{2 t} \geq 2 m$, and since $0 \leq \phi \leq 1$ this allows us to replace the power on $\phi$ in the first term on the right with $2 m$, and hence we obtain

$$
\int \omega_{1} e^{2 t u} \phi^{2 m-2}|\nabla \phi|^{2} \leq\left(\int \omega_{2} e^{(2 t+1) u} \phi^{2 m} d x\right)^{\frac{2 t}{2 t+1}}\left(\int \frac{\omega_{1}^{2 t+1}}{\omega_{2}^{2 t}}|\nabla \phi|^{2(2 t+1)}\right)^{\frac{1}{2 t+1}} .
$$

We now take the test functions $\phi$ to be such that $0 \leq \phi \leq 1$ with $\phi$ supported in the ball $B_{2 R}$ with $\phi=1$ on $B_{R}$ and $|\nabla \phi| \leq \frac{C}{R}$, where $C>0$ is independent of $R$. Using this choice of $\phi$ we obtain

$$
\int \omega_{1} e^{2 t u} \phi^{2 m-2}|\nabla \phi|^{2} \leq\left(\int \omega_{2} e^{(2 t+1) u} \phi^{2 m}\right)^{\frac{2 t}{2 t+1}} I_{G}^{\frac{1}{2 t+1}} .
$$

One similarly shows that

$$
\int \omega_{1} e^{2 t u} \phi^{2 m-1}|\Delta \phi| \leq\left(\int \omega_{2} e^{(2 t+1) u} \phi^{2 m}\right)^{\frac{2 t}{2 t+1}} I_{G}^{\frac{1}{2 t+1}} .
$$

So, combining the results we obtain

$$
\begin{aligned}
\frac{(2-t)}{2} \int \omega_{2} e^{(2 t+1) u} \phi^{2 m} \leq & C_{m}\left(\int \omega_{2} e^{(2 t+1) u} \phi^{2 m} d x\right)^{\frac{2 t}{2 t+1}} I_{G}^{\frac{1}{2 t+1}} \\
& -D_{m} \int e^{2 t u} \phi^{2 m-1} \nabla \omega_{1} \cdot \nabla \phi
\end{aligned}
$$

We now estimate this last term. A similar argument using Hölder's inequality shows that

$$
\int e^{2 t u} \phi^{2 m-1}\left|\nabla \omega_{1}\right||\nabla \phi| \leq\left(\int \omega_{2} \phi^{2 m} e^{(2 t+1) u} d x\right)^{\frac{2 t}{2 t+1}} J_{G}^{\frac{1}{2 t+1}} .
$$

Combining the results gives that

$$
(2-t)\left(\int \omega_{2} e^{(2 t+1) u} \phi^{2 m} d x\right)^{\frac{1}{2 t+1}} \leq I_{G}^{\frac{1}{2 t+1}}+J_{G}^{\frac{1}{2 t+1}}
$$

and now we send $R \rightarrow \infty$ and use the fact that $I_{G}, J_{G} \rightarrow 0$ as $R \rightarrow \infty$ to see that

$$
\int \omega_{2} e^{(2 t+1) u}=0
$$

which is clearly a contradiction. Hence there is no stable sub-solution of $(G)$. 
(2) Suppose that $u>0$ is a stable sub-solution (super-solution) of $(L)$. Then a similar calculation as in (1) shows that for $p-\sqrt{p(p-1)}<t<p+\sqrt{p(p-1)}$ $\left(0<t<\frac{1}{2}\right)$ one has

$$
\begin{aligned}
\left(p-\frac{t^{2}}{2 t-1}\right) \int \omega_{2} u^{2 t+p-1} \phi^{2 m} \leq & D_{m} \int \omega_{1} u^{2 t} \phi^{2(m-1)}\left(|\nabla \phi|^{2}+\phi|\Delta \phi|\right) \\
& +C_{m} \frac{(1-t)}{2(2 t-1)} \int u^{2 t} \phi^{2 m-1} \nabla \omega_{1} \cdot \nabla \phi .
\end{aligned}
$$

One now applies Hölder's argument as in (1), but the terms $I_{L}$ and $J_{L}$ will appear on the right-hand side of the resulting equation. This shift from a sub-solution to a super-solution depending on whether $t>\frac{1}{2}$ or $t<\frac{1}{2}$ is a result from the sign change of $2 t-1$ at $t=\frac{1}{2}$. We leave the details for the reader.

(3) This case is also similar to (1) and (2).

Proof of Theorem 1.2. (1) Again we suppose that there is a stable sub-solution $u$ of $(G)$. Our starting point is (2.2), and we wish to be able to drop the term

$$
-D_{m} \int e^{2 t u} \phi^{2 m-1} \nabla \omega_{1} \cdot \nabla \phi
$$

from (2.2). We can choose $\phi$ as in the proof of Theorem 1.1 but also such that $\nabla \phi(x)=-C(x) x$, where $C(x) \geq 0$. So if we assume that $\nabla \omega_{1} \cdot x \leq 0$ for large $x$, then we see that this last term is non-positive and hence we can drop the term. Then the proof is as before, but now we only require that $\lim _{R \rightarrow \infty} I_{G}=0$.

(2) Suppose that $u>0$ is a stable sub-solution of $(L)$ and so (2.7) holds for all $p-\sqrt{p(p-1)}<t<p+\sqrt{p(p-1)}$. Now we wish to use monotonicity to drop the term from (2.7) involving the term $\nabla \omega_{1} \cdot \nabla \phi . \phi$ is chosen similarly as in (1), but here one notes that the coefficient for this term changes sign at $t=1$, and hence by restriction of $t$ to the appropriate side of (1) (along with the above condition on $t$ and $\omega_{1}$ ) we can drop the last term depending on which monotonicity we have and hence obtain a contraction we only require that $\lim _{R \rightarrow \infty} I_{L}=0$. The result for the non-existence of a stable super-solution is similar, but here one uses the restriction $0<t<\frac{1}{2}$.

(3) The proof here is similar to (1) and (2), and we omit the details.

Proof of Corollary 1. We suppose that $\omega_{1} \leq C \omega_{2}$ for large $x, \omega_{2} \in L^{\infty}, \nabla \omega_{1}(x) \cdot x \leq$ 0 for large $x$.

(1). Since $\nabla \omega_{1} \cdot x \leq 0$ for large $x$ we can apply Theorem 1.2 to show the nonexistence of a stable solution to $(G)$. Note that with the above assumptions on $\omega_{i}$ we have that

$$
I_{G} \leq \frac{C R^{N}}{R^{4 t+2}}
$$

For $N \leq 9$ we can take $0<t<2$ but close enough to 2 so that the right-hand side goes to zero as $R \rightarrow \infty$.

Both (2) and (3) also follow directly from applying Theorem 1.2, Note that one can say more about (2) by taking the multiple cases as listed in Theorem 1.2 , but we have chosen to leave this to the reader.

Proof of Corollary 2. Since we have no monotonicity conditions now we will need both $I$ and $J$ to go to zero to show the non-existence of a stable solution. Again 
the results are obtained immediately by applying Theorem 1.1, and we prefer to omit the details.

Proof of Theorem 1.3. (1) If $N+\alpha-2<0$, then using Remark 1 one easily sees there is no stable sub-solution of $(G)$ and $(L)$ (positive for $(L)$ ) or a positive stable super-solution of $(M)$. So we now assume that $N+\alpha-2>0$. Note that the monotonicity of $\omega_{1}$ changes when $\alpha$ changes sign, and hence one would think that we need to consider separate cases if we hope to utilize the monotonicity results. But a computation shows that in fact $I$ and $J$ are just multiples of each other in all three cases, so it suffices to show, say, that $\lim _{R \rightarrow \infty} I=0$.

(2) Note that for $R>1$ one has

$$
\begin{aligned}
I_{G} & \leq \frac{C}{R^{4 t+2}} \int_{R<|x|<2 R}|x|^{\alpha(2 t+1)-2 t \beta} \\
& \leq \frac{C}{R^{4 t+2}} R^{N+\alpha(2 t+1)-2 t \beta},
\end{aligned}
$$

and so to show the non-existence we want to find some $0<t<2$ such that $4 t+2>N+\alpha(2 t+1)-2 t \beta$, which is equivalent to $2 t(\beta-\alpha+2)>(N+\alpha-2)$. Now recall that we are assuming that $0<N+\alpha-2<4(\beta-\alpha+2)$ and hence we have the desired result by taking $t<2$ but sufficiently close. The proof of the non-existence results for (3) and (4) are similar and we omit the details.

(5) We now assume that $N+\alpha-2>0$. In showing the existence of stable sub/super-solutions we need to consider $\beta-\alpha+2<0$ and $\beta-\alpha+2>0$ separately.

- $(\beta-\alpha+2<0)$. Here we take $u(x)=0$ in the case of $(G)$ and $u=1$ in the case of $(L)$ and $(M)$. In addition we take $g(x)=\varepsilon$. It is clear that in all cases $u$ is the appropriate sub- or super-solution. The only thing one needs to check is the stability. In all cases this reduces to trying to show that we have

$$
\sigma \int\left(1+|x|^{2}\right)^{\frac{\alpha}{2}-1} \phi^{2} \leq \int\left(1+|x|^{2}\right)^{\frac{\alpha}{2}}|\nabla \phi|^{2},
$$

for all $\phi \in C_{c}^{\infty}$, where $\sigma$ is some small positive constant; it is either $\varepsilon$ or $p \varepsilon$ depending on which equation we are examining. To show this we use the result from Corollary 3 and we drop a few positive terms to arrive at

$$
\int\left(1+|x|^{2}\right)^{\frac{\alpha}{2}}|\nabla \phi|^{2} \geq\left(t+\frac{\alpha}{2}\right) \int\left(N-2(t+1) \frac{|x|^{2}}{1+|x|^{2}}\right)\left(1+|x|^{2}\right)^{-1+\frac{\alpha}{2}},
$$

which holds for all $\phi \in C_{c}^{\infty}$ and $t, \alpha \in \mathbb{R}$. Now, since $N+\alpha-2>0$, we can choose $t$ such that $-\frac{\alpha}{2}<t<\frac{n-2}{2}$. So, the integrand function on the right-hand side is positive, and since for small enough $\sigma$ we have

$$
\sigma \leq\left(t+\frac{\alpha}{2}\right)\left(N-2(t+1) \frac{|x|^{2}}{1+|x|^{2}}\right) \quad \text { for all } x \in \mathbb{R}^{N}
$$

we get stability.

- $(\beta-\alpha+2>0)$. In the case of $(G)$ we take $u(x)=-\frac{\beta-\alpha+2}{2} \ln \left(1+|x|^{2}\right)$ and $g(x):=(\beta-\alpha+2)\left(N+(\alpha-2) \frac{|x|^{2}}{1+|x|^{2}}\right)$. By a computation one sees that $u$ is a sub-solution of $(G)$, and hence we now need only to show the stability, which amounts to showing that

$$
\int \frac{g(x) \psi^{2}}{\left(1+|x|^{2}\right)^{-\frac{\alpha}{2}+1}} \leq \int \frac{|\nabla \psi|^{2}}{\left(1+|x|^{2}\right)^{-\frac{\alpha}{2}}},
$$


for all $\psi \in C_{c}^{\infty}$. To show this we use Corollary [3. So we need to choose an appropriate $t$ in $-\frac{\alpha}{2} \leq t \leq \frac{N-2}{2}$ such that for all $x \in \mathbb{R}^{N}$ we have

$$
\begin{aligned}
(\beta-\alpha+2)\left(N+(\alpha-2) \frac{|x|^{2}}{1+|x|^{2}}\right) \leq & \left(t+\frac{\alpha}{2}\right)^{2} \frac{|x|^{2}}{\left(1+|x|^{2}\right.} \\
& +\left(t+\frac{\alpha}{2}\right)\left(N-2(t+1) \frac{|x|^{2}}{1+|x|^{2}}\right) .
\end{aligned}
$$

With a simple calculation one sees that we just need to have

$$
\begin{aligned}
(\beta-\alpha+2) & \leq\left(t+\frac{\alpha}{2}\right) \\
(\beta-\alpha+2)(N+\alpha-2) & \left.\leq\left(t+\frac{\alpha}{2}\right)\left(N-t-2+\frac{\alpha}{2}\right)\right) .
\end{aligned}
$$

If one takes $t=\frac{N-2}{2}$ in the case where $N \neq 2$ and $t$ close to zero in the case for $N=2$, one easily sees that the above inequalities both hold after considering all the constraints on $\alpha, \beta$ and $N$.

We now consider the case of $(L)$. Here one takes $g(x):=\frac{\beta-\alpha+2}{p-1}(N+$ $\left.\left(\alpha-2-\frac{\beta-\alpha+2}{p-1}\right) \frac{|x|^{2}}{1+|x|^{2}}\right)$ and $u(x)=\left(1+|x|^{2}\right)^{-\frac{\beta-\alpha+2}{2(p-1)}}$. Using essentially the same approach as in $(G)$ one shows that $u$ is a stable sub-solution of $(L)$ with this choice of $g$.

For the case of $(M)$ we take $u(x)=\left(1+|x|^{2}\right)^{\frac{\beta-\alpha+2}{2(p+1)}}$ and $g(x):=\frac{\beta-\alpha+2}{p+1}(N+$ $\left.\left(\alpha-2+\frac{\beta-\alpha+2}{p+1}\right) \frac{|x|^{2}}{1+|x|^{2}}\right)$.

\section{REFERENCES}

[1] D. Castorina, P. Esposito and B. Sciunzi; Low dimensional instability for semilinear and quasilinear problems in $\mathbb{R}^{N}$. Commun. Pure Appl. Anal. 8 (2009), no. 6, 1779-1793. MR2552149(2010m:35172)

[2] C. Cowan; Optimal Hardy inequalities for general elliptic operators with improvements, Commun. Pure Appl. Anal. 9 (2010), no. 1, 109-140. MR2556749 (2010m:35008)

[3] E. N. Dancer; Stable and finite Morse index solutions on $\mathbb{R}^{n}$ or on bounded domains with small diffusion. Trans. Amer. Math. Soc. 357 (2005), 1225-1243. MR2110438(2006e:35093)

[4] L. Dupaigne and A. Farina; Stable solutions of $-\Delta u=f(u)$ in $\mathbb{R}^{N}$. J. Eur. Math. Soc. (JEMS) 12 (2010), no. 4, 855-882. MR.2654082 (2011f:35088)

[5] P. Esposito; Linear instability of entire solutions for a class of non-autonomous elliptic equations. Proc. Roy. Soc. Edinburgh Sect. A 138 (2008), no. 5, 1005-1018. MR2477449 (2010b:35173)

[6] P. Esposito; Compactness of a nonlinear eigenvalue problem with a singular nonlinearity. Commun. Contemp. Math. 10 (2008), no. 1, 17-45. MR2387858 (2009j:35086)

[7] P. Esposito, N. Ghoussoub, and Y. Guo; Mathematical analysis of partial differential equations modeling electrostatic MEMS, Courant Lecture Notes in Mathematics, 20. Courant Institute of Mathematical Sciences, New York; American Mathematical Society, Providence, RI, 2010. MR2604963(2011c:35005)

[8] P. Esposito, N. Ghoussoub, and Y. Guo; Compactness along the branch of semistable and unstable solutions for an elliptic problem with a singular nonlinearity. Comm. Pure Appl. Math. 60 (2007), no. 12, 1731-1768. MR2358647 (2009c:35119)

[9] A. Farina; On the classification of solutions of the Lane-Emden equation on unbounded domains of $\mathbb{R}^{N}$, J. Math. Pures Appl. (9) 87 (2007), no. 5, 537-561. MR2322150(2008c:35070)

[10] A. Farina; Stable solutions of $-\Delta u=e^{u}$ on $\mathbb{R}^{N}$, C. R. Math. Acad. Sci. Paris 345 (2007), no. 2, 63-66. MR2343553(2008e:35063) 
[11] L. Ma and J. C. Wei; Properties of positive solutions to an elliptic equation with negative exponent. J. Funct. Anal. 254 (2008), no. 4, 1058-1087. MR2381203 (2009b:35122)

[12] W. M. Ni; A nonlinear Dirichlet problem on the unit ball and its applications. Indiana Univ. Math. J. 31 (1982), no. 6, 801-807. MR674869(84b:35051)

Department of Mathematics, Stanford University, Stanford, California 94305

E-mail address: ctcowan@stanford.edu

Department of Mathematics, University of British Columbia, Vancouver, B.C., CANAdA V6T 1 Z2

E-mail address: fazly@math.ubc.ca 